\title{
Modeling Long-Term Signature Validation for Resolution of Dispute
}

\author{
Moez Ben MBarka ${ }^{1,2}$, Francine Krief ${ }^{2}$, and Olivier Ly ${ }^{2}$ \\ 1 Cryptolog International, Paris, France \\ moez. benmbarka@cryptolog.com \\ 2 LaBRI, University of Bordeaux 1, 33400 Talence, France \\ \{krief, ly\}@labri.fr
}

\begin{abstract}
This paper considers the case where a dispute occurs between a verifier and a signer about the validity of a digital signature. In non-repudiation services such dispute may occur long after the signature creation and approval. We present a security model for digital signature validation with the notion of dispute. The first contribution of this paper is the definition of the semantics of a Resolution of Dispute Rule (RDR) in the scope of this model. The second contribution is a calculus for reasoning about the validation of digital signatures at a particular date which may be in the past (so-called long-term signature validation). This calculus is then used to implement the RDR. The usefulness of the calculus is demonstrated through modeling Evidence Record Syntax (ERS), one of the main protocols used in practice for long-term signature validation.
\end{abstract}

Keywords: long-term signature validation, resolution of dispute, formal calculus, Public Key Infrastructure.

\section{Introduction}

A digital signature is a piece of digital data that can be used to guarantee integrity, authenticity, and non-repudiation in electronic communications. It is nowadays based on public key cryptography. To see how it works, suppose that a signer Bob wants to sign a message that will be verified by Alice. Bob computes the hash of the message and then encrypts the output using his private key. Alice can then verify the signature using Bob's public key. This mathematical verification allows to prove the integrity of the message and the binding between the message and a public key. Authenticity and non repudiation come from verifying the binding between this public key and the signer using public key certificates. In the widely used X.509-based PKI model (PKIX [1]), this binding is signed by a Certification Authority (CA) which is trusted to verify the identity of the certificate's owner (called certificate's subject) before signing the certificate. The certificate's owner may be another CA (certification authority certificate) or an entity which is not a CA (end entity certificate). Various circumstances (e.g. key compromise) may cause a certificate to become invalid prior to its expiration. Under such circumstances, the CA needs to revoke the certificate [1,2]. 


\subsection{Statement of the Problem}

Digital signatures are gradually acquiring legal equivalence of handwritten signatures promoting their use to secure business and commercial digital communications. Nowadays digital signature schemes and the infrastructure within which they operate have time limitations which may be due to some inner expiration dates (e.g. the expiration of a certificate) or to the fact that the underlying cryptographic security relies on the presumed computational difficulty of certain theoretic problems (e.g. integer factorization) [3]. This situation is disturbing considering that there are many cases such as government records where the signatures are required by civil laws to be kept valid for a long period of time.

We address this issue by modeling long-term signature validation in the scope of a dispute between the typical actors: a verifier (Alice) who claims the validity of the signature at some date (possibly in the past) and a signer (Bob) who denies this claim. Imagine for a moment a judge trying to resolve a dispute between Alice and Bob in the year 2030. To prove their point, the parties invoke several records that were digitally signed by them in the year 2005. The judge needs to decide if the evidence presented is admissible in court. We use our model to define the semantics of a resolution of dispute rule that can be used by the judge to take a decision about the validity of the presented digital signatures.

\subsection{Related Work}

Long-Term Validation. It is already known that time-stamp services can be used to maintain digital document (in particular digital signatures) integrity and authenticity. The time-stamp is a digital attestation signed by a trusted third party, called Time-Stamping Authority (TSA), that a submitted document has been presented to the TSA at a certain time. For a document $m$, the time-stamp is a signature where the signer is a TSA and the signed message includes the pair $(T, h(m)))$ such as $T$ is the current time retrieved from a reliable source of time and $h$ is a cryptographic hash function.

Many technical frameworks for long-term signatures validation are defined in the literature $4,5,6,7,8$, and are mainly based on time-stamping: the signature is time-stamped indicating that it was created at a moment before a subsequent key or algorithm compromise; this time-stamp is refreshed before the used algorithms or keys become compromised, ensuring its validity through the years. Most of these techniques rely on the use of Evidence Record Syntax (ERS) [8]. ERS uses a chain of time-stamps to prove the existence of some digital data at a certain date. Each time-stamp may protect a single data object or to a group of objects using a hash tree where only the root of the tree is time-stamped. ERS defines two renewal algorithms: the simple and the complex renewal methods. In the case of the simple renewal, only the last generated time-stamp has to be timestamped again. In this scenario, it is not necessary to access the initially archived data objects themselves, since the algorithms used for the previous time-stamps are recognized as being still reliable. The simple renewal method is not sufficient when the hash functions used in the previous time-stamps become insecure. In 
this scenario, not only the initial time-stamps but also the initial data have to be time-stamped again using newer algorithms.

PKI Calculus. In 9, Maurer presented the pioneer deterministic calculus for PKI. The calculus is based on four statements (authenticity, trust, certificate, recommendation) and two inference rules. Starting from an initial set of statements (called Alice's initial view), Alice uses the inference rules to derive new statements. In this model, Alice can use a certificate issued by a CA $X$ for a user Bob if and only if she can derive the following statements: she has a certificate signed by $X$ for Bob, she knows $X$ 's public key and believes that it is authentic and she trusts $X$ to correctly authenticate the owner of a public key. The objective of Alice is to derive these statements from her initial view using the inference rules. Later, many authors [10,11] revisited this model to add additional concepts such as time, certificate expiration and revocation.

Signature Validity Models. In [12, the authors surveyed three validity models for digital signatures:

1. Shell model: in this model, the signature is always validated at the current date. The signature becomes invalid once the signer certificate gets expired.

2. Modified shell model: The signature is validated at the signing date and not at the verification date. Therefore, the expiration or revocation of the signer certificate does not affect the signature validity.

3. Chain model: in addition to the signing date, this model takes into account the issuance date of each certificate in the certificate validation chain. Not only, the expiration of the signer certificate does not affect the signature validity but also the signature remains valid even if an intermediate $\mathrm{CA}$ is revoked before the signing date.

The natural conclusions from these models are that:

- the shell model is only suitable for authentication services where the validity of the signature is required for short term.

- the modified shell and the chain models have the fundamental property: if a digital signature is valid at the signing date, it remains valid forever. Therefore, these models are suitable for long-term validation.

\subsection{Motivations and Organization of the Paper}

While many long-term signature validation frameworks [4,5, 6, and standards [8, 7] are currently in use in practice, to the best of our knowledge, there is no formal model showing that they provide the evidences they claim. These frameworks miss formal definitions of long-term validation semantics. Defining such semantics is necessary to have a formal calculus. This is the first contribution of this paper. On the other hand, Maurer's calculus and its extensions showed above can be used to reason about public key authenticity but miss many features required to deal with long-term validation. The second contribution is the proposition of a formal calculus which captures these features (Figure 1). 


\begin{tabular}{|c|c|c|}
\hline Feature & our calculus & Maurer's calculus and extensions \\
\hline Authenticity and revocation & yes & yes \\
\hline Time aware signature validation & yes & no \\
\hline Algorithm security & yes & no \\
\hline Proof of existence & yes & no \\
\hline
\end{tabular}

Fig. 1. Our calculus vs previous calculus based on Maurer's model

The rest of the paper is organized as follows: Section 2 presents a simple security model for digital signature validation. Section 3 defines the resolution of dispute in the scope of this model. Section 4 presents our calculus which is used to implement the Resolution of Dispute Rule in Section 5. Section 6 uses the calculus to model ERS. Finally, Section 7 concludes the paper.

\section{Security Model and Notations}

In our model, a digital signature is denoted $\operatorname{Sig}(X, m, s a, s v)$ where $X$ identifies the signer, $m$ is the signed message, $s a$ identifies the signature algorithm, and $s v$ is the signature value computed on $m$ using $s a$ and the private key corresponding to $X$ 's public key. The integrity and authenticity come from the cryptographic properties of the signature algorithm sa (namely, existential unforgeability against chosen message attacks [13]). A signature is said "cryptographically valid" if and only if $s v$ verifies with $X$ 's public key on the message $m$ using the signature algorithm $s a$. Non-repudiation relies on the authenticity of the signer's public key that can be derived from the certificate. The most widely PKI trust model is a hierarchy of CAs. In this model, the validation of a certificate at some date $T$ consists in building and validating a certificate path from a trusted CA (called trust anchor) to the certificate to validate. Below, we combine the certificate validation algorithm defined in PKIX [1] with the cryptographic verification to define a Signature Validation Function $(S V F)$. In the model, we accept SVF as the mean to prove integrity, authenticity and non-repudiation.

Definition 1 (Signature Validation Function (SVF)). SVF takes as input the following: a signature $\operatorname{Sig}(X, m, s a, s v)$, a set of trust anchors $\mathcal{K}$, a set of validation objects $\mathcal{O}$, and a date $T$. The function returns a pair (status, $\mathcal{P}$ ) such that status $=$ true if and only if:

- the signature is cryptographically valid.

- there exists a sequence of certificates Cert $_{1}, \ldots$, Cert $_{n}$ in $\mathcal{O}$ such that:

- $\forall i \in[1, n-1]$, Cert $_{i}$ 's subject is Cert $_{i+1}$ 's issuer and Cert ${ }_{i}$ is a certification authority certificate.

- Cert $_{1}$ is issued by a trust anchor in $\mathcal{K}$.

- Cert $_{n}$ is an end entity certificate and is issued to X.

- $\forall i \in[1, n]$, Cert $_{i}$ is valid at $T$ and there exists a revocation object Rev R $_{i}$ $\mathcal{O}$ signed by Cert $_{i}$ 's issuer indicating that Cert $_{i}$ is not revoked at $T$. 
- $\forall i \in[1, n]$, the signatures in Cert $_{i}$ and Rev R $_{i}$ are cryptographically valid.

If status = true then the signature is said "valid" at $T$ and $\mathcal{P}$ is the sequence $\left\{\operatorname{Cert}_{1}, \operatorname{Rev}_{1}, \ldots, \operatorname{Cert}_{n}, \operatorname{Rev}_{n}\right\}$. Otherwise, the signature said is "invalid" at $T$.

The function above requires a set of trust anchors that the verifier trusts without prior verification. In addition, the verifier needs to trust some assumptions about the security of the used algorithms. This yields a particular set that we will call Initial View of Trust $(\mathcal{I} \mathcal{V})$ :

Definition 2 (Initial View of Trust $(\mathcal{I V} \mathcal{T}(T))$ ). $\mathcal{I V} \mathcal{T}(T)$ is composed of the following:

- a set of trust anchors $\mathcal{K}=\left\{T A_{1}, \ldots, T A_{n}\right\}$ such that $\forall i \in[1, n], T A_{i}$ is a $C A$ identified by a certification authority certificate.

- a set of algorithm trust assertions $\mathcal{A}=\left\{\left(a_{1}, T_{a_{1}}\right), \ldots,\left(a_{k}, T_{a_{k}}\right)\right\}$, such that $\forall i \in[1, k], a_{i}$ is a cryptographic hash function or a signature algorithm considered secure until at least the date $T_{a_{i}}$.

The semantics of "secure" depends on the nature of the cryptographic algorithm. In our model, we will say that an algorithm is secure until at least $T$ if it is commonly believed that the scientific knowledge and computing power will not allow to break the security properties of the algorithm until at least $T$.

\section{Resolution of Dispute}

In the model, Bob uses his private key to sign some digital data and Alice verifies the signature using Bob's public key. For instance, consider a scenario where Bob signs a contract with Alice to pay monthly some amount of money. If in the future, Bob's certificate is revoked, he may claim that he never signed the contract and stop paying Alice. This leads to a dispute situation: on one hand, Alice claims the validity of a signature and that this signature has been created at a date in the past (before revocation); on the other hand, Bob denies these claims. First, we define the dispute. Next, we define the resolution of dispute.

Definition 3 (Dispute). If Alice (A) claims at a date $T \geq T_{d}$ that a signature $\operatorname{Sig}(B, m, s a, s v)$ is signed by $B o b(B)$ and is valid at $T_{d}$; and if Bob denies any of these claims, we say that Alice is in a dispute Dispute $\left(A, B, S, T_{d}\right)$ with Bob. $T_{d}$ is called the dispute date and $T$ the resolution date.

Both Alice and Bob rely on a third party, called the Judge, to resolve the dispute. The Judge can resolve the dispute using a Resolution of Dispute Rule (RDR) at $T$ based on validation objects (certificates, CRLs...) provided by Alice:

Definition 4 (Resolution of Dispute Rule). If a dispute Dispute $\left(A, B, S, T_{d}\right)$ occurs between Alice and Bob at T, the Judge executes the RDR. The RDR takes as input the following: the signature $S$, a set of validation objects $\mathcal{O}$ provided by Alice, $\mathcal{I V T}(T)$, the dispute date $T_{d}$, and the resolution date $T$. It outputs a pair 
(status, $\mathcal{P})$ such that status $\in\{$ true, false $\}$ and $\mathcal{P} \subseteq\{S\} \cup \mathcal{O} \cup \mathcal{I} \mathcal{V} \mathcal{T}(T)$. If status $=$ true, the Judge rules in favor of Alice. Otherwise, he rules in favor of Bob. In addition, if status $=$ true then $\mathcal{P} \neq \emptyset$ is the proof of the validity of the claims of Alice and is called the judgment proof.

\subsection{Threat Model}

The Judge is trusted to honestly run the RDR and honestly output the result. However, any of the other actors can run the RDR and thus verify the output. Indeed, the Judge can be seen as a procedure (e.g. enforced by a court) whose output can be verified by other actors.

$\mathcal{I V T}$ is a public set trusted by all actors. This assumes that no attacker has significantly more computing power or cryptanalytic knowledge than what is widely known. $\mathcal{I} \mathcal{V} \mathcal{T}$ may naturally be updated to take account of new algorithms and authorities. We will assume that any update to $\mathcal{I} \mathcal{V} \mathcal{T}$ does not remove or reduce the validity interval of any algorithm or authority in the previous $\mathcal{I} \mathcal{V} \mathcal{T}$.

Finally, a traditional assumption in a PKI context is that honest involved authorities (namely TSAs and CAs) act honestly. In particular, we assume that the CAs conform to their Certification Policies (CP) that describe certificate issuance and revocation rules. We assume that the CPs of all involved CAs guarantee at least the following: a certificate can be revoked but not suspended and a signer can not access the signing key before the issuance date of her certificate. We will also assume that all honest CAs provide instant revocation for all honestly issued certificates during their validity intervals: there is no delay between effective compromise and the publication of the revocation information. We are aware that in the real world there is an inevitable delay between the compromise and the revocation. For the sake of simplicity, this delay will be first ignored but will be discussed later.

\subsection{RDR Semantics}

The objective of RDR is to build proofs of the validity of Alice's claims at the resolution date. If such proofs can be built in a verifiable way, the Judge rules in favor of Alice, otherwise, he rules in favor of Bob. Note that the latter case does not mean that Alice's claims are provably false, but only that these claims can not be proven using the provided inputs. We start by defining two properties that must be satisfied by the RDR. The first requirement is Correctness. This means that if the Judge rules in favor of Alice then her claims are correct. Naturally, we use the modified shell model: the signature is validated at the signing date.

Property 1 (Correctness). Given the the signature $S_{d}=\operatorname{Sig}(B, m, s a, s v)$ and the Dispute $\left(A, B, S_{d}, T_{d}\right)$; if $\operatorname{RDR}\left(S_{d}, \mathcal{O}, \mathcal{I} \mathcal{V} \mathcal{T}(T), T_{d}, T\right)$ outputs true then $S_{d}$ is created at a date $T_{s} \leq T_{d}$ and is valid at $T_{s}$

The second consideration is that a digital signature can not be repudiated. In other words, If a signature is accepted valid at some date is should remain valid 
forever: if a Judge rules in favor of the validity of a signature at a date $T_{d}$, he would rule in favor of the validity of the signature at any date after the date for which he has a proof of the existence and unforgeability of the signature.

Property 2 (Eternal Validity). Given the signature $S_{d}=\operatorname{Sig}(B, m, s a, s v)$ and the dispute Dispute $\left(A, B, S_{d}, T_{d}\right)$; if $\operatorname{RDR}\left(S_{d}, \mathcal{O}, \mathcal{I} \mathcal{V} \mathcal{T}(T), T_{d}, T\right)$ outputs true and $S_{d}$ is proven to exist at $T_{s}$ and uses an algorithm trusted to be secure until at least $T_{s}$ such that $T_{s} \leq T_{d}$, then $\operatorname{RDR}\left(S_{d}, \mathcal{O}, \mathcal{I} \mathcal{V} \mathcal{T}(T), T_{d}^{\prime}, T\right)$ outputs true $\forall T_{d}^{\prime} \geq T_{s}$.

Now let go to the definition of the RDR semantics:

Definition 5 (RDR Semantics). Given the signature $S_{d}=\operatorname{Sig}(B, m, s a, s v)$, the set of the validation objects $\mathcal{O}$ provided by Alice and the Dispute $\left(A, B, S_{d}, T_{d}\right)$; $\operatorname{RDR}\left(S_{d}, \mathcal{O}, \mathcal{I} \mathcal{V}(T), T_{d}, T\right)$ outputs true if and only if there exists a date $T_{s} \leq T_{d}$ such that the Judge can prove that all the following statements are valid at $T$ :

1. there exists a date $T_{x} \geq T_{s}$ such that $\operatorname{SVF}\left(S_{d}, \mathcal{K}, \mathcal{O}, T_{x}\right)=($ true, $\mathcal{P})$.

2. each validation object $V$ in $\mathcal{P}$ exists at a date $T_{V} \leq T_{x}$ and uses an algorithm trusted to be secure until at least $T_{V}$.

3. $S_{d}$ exists at $T_{s}$ and uses an algorithm trusted to be secure until at least $T_{s} . \circ$

Proposition 1. The RDR Semantics has both the Correctness and Eternal Validity properties.

Proof.

- Correctness:The first part of the correctness is obvious: since $S_{d}$ exists at $T_{s}$, then $S_{d}$ is created at a date $T_{s}^{\prime} \leq T_{s} \leq T_{d}$. It remains to prove that $S_{d}$ is valid at $T_{s}^{\prime}$. Let us denote $\mathcal{I} \mathcal{V} \mathcal{T}(T)=(\mathcal{K}, \mathcal{A})$ and $\mathcal{P}=\left\{\right.$ Cert $_{1}$, Rev $_{1}, \ldots$, Cert $_{n}$, Rev $\left._{n}\right\}$ the sequence of certificates and revocation objects returned by SVF at $T_{x}$ and $\left\{C A_{0}, \ldots, C A_{n-1}\right\}$ the set of CAs such that $\forall i \in[1, n-1], C A_{i}$ is the CA identified in $C_{e r t}$, and $C A_{0}$ is the trust anchor from $\mathcal{K}$ that issued Cert $_{1}$. $\forall i$, we will denote $\left[T_{b_{i}}, T_{e_{i}}\right]$ the validity interval of $C_{e r t}$. First, we show by induction that $\forall i \in[1, n]$, Cert $_{i}$ and $\operatorname{Rev}_{i}$ are honestly generated.

- for $i=1$, Cert $_{1}$ and $R e v_{1}$ are honestly generated by $C A_{0}$ because they are created, using a secure algorithm, by a trust anchor which can not be compromised.

- assume that Cert $_{i}$ and $R e v_{i}$ are honestly generated. If $C_{e r t} t_{i+1}$ or $\operatorname{Rev}_{i+1}$ is not honestly generated but forged (say by Alice), then Alice needs to access to the signing key (because Cert $_{i+1}$ and $R e v_{i+1}$ are created at date before $T_{x}$ using a secure algorithm). So, we have two cases:

* The private key of $C A_{i}$ is compromised (anyone including Alice may have access to this private key). So, $\operatorname{Rev}_{i}$ is also forged (because it indicates that Cert $_{i}$ is not revoked at $T_{x}$ ).

* $C A_{i}$ is not an honest CA. So Cert $t_{i}$ is forged.

Then, either Cert $_{i}$ or $R e v_{i}$ is forged which contradicts the induction hypothesis. Hence, $\operatorname{Cert}_{i+1}$ and $\operatorname{Rev}_{i+1}$ are honestly generated. Thus, $\forall i \in[1, n]$, Cert $_{i}$ and $\operatorname{Rev}_{i}$ are honestly generated by honest CAs. 
On the other hand, a private key is not issued before the certificate issuance date. Since the creation of the signature at $T_{s}^{\prime}$ needs the private key (the signature algorithm is secure at $\left.T_{s}^{\prime} \leq T_{s}\right)$, this gives $T_{s}^{\prime} \in\left[T_{b_{n}}, T_{d}\right]$ and therefore $\forall i \in[1, n], T_{s}^{\prime} \in\left[T_{b_{i}}, T_{e_{i}}\right]$. Since Cert $_{i}$ is not revoked at $T_{x}$, then it is not revoked at any date in $\left[T_{b_{i}}, T_{x}\right]$ (a certificate can not be suspended) and in particular at $T_{s}^{\prime}$. In addition, since $\forall i \in[1, n], \operatorname{Cert}_{i}$ is honestly issued by an honest $\mathrm{CA}$, there exists a revocation object $R e v_{i}^{\prime}$ issued by $C A_{i-1}$ that indicates that Cert $_{i}$ is not revoked at $T_{s}^{\prime}$. Thus, the sequence $\mathcal{P}^{\prime}=\left\{\operatorname{Cert}_{1}, \operatorname{Rev}_{1}^{\prime}, \ldots, \operatorname{Cert}_{n}=B, \operatorname{Rev}_{n}^{\prime}\right\}$ verifies the conditions of $S V F$ and then $S V F\left(S, \mathcal{K}, \mathcal{P}^{\prime}, T_{s}^{\prime}\right)$ outputs true. Hence, the Correctness property.

- Eternal validity:If it happens that $S_{d}$ is proven to exist at $T_{s}$ and uses an algorithm trusted to be secure until at least $T_{s}$ and $\operatorname{RDR}\left(S_{d}, \mathcal{O}, \mathcal{I} \mathcal{V} \mathcal{T}(T), T_{d_{1}}, T\right)$ outputs true such that $T_{d_{1}} \geq T_{s}$, then there exists a date $T_{x} \geq T_{s}$ which verifies the first statements and a set of $n$ dates $T_{V O_{1}} \ldots T_{V O_{n}}$ which verify the second statements. We can use the same dates for any date $T_{d_{2}} \geq T_{s}$ such that $\operatorname{RDR}\left(S_{d}, \mathcal{O}, \mathcal{I} \mathcal{V}(T), T_{d_{2}}, T\right)$ outputs also true.

\subsection{Discussions}

Role of Bob. Bob is not involved in the semantics above. One may think that this is unfair towards Bob. Due to the Correctness property, if it happens that the Judge rules in favor of Alice although Bob is not the actual signer, then this means that Bob's private key has been compromised before the date $T_{s}$ but the revocation information was not yet published at $T_{x}$. This can not happen in the considered model as we assumed instant revocation. However, in the real world, there is always an inevitable delay between the effective compromise and the publication of the revocation information [14. Therefore, it is important to wait some period of time after the signing date before considering the signature valid. This period (called caution period) is the estimated time duration required to process and publish the last revocation information. The value of the caution period must be established in agreement between all actors.

Using a Caution Period $\boldsymbol{C}$. To consider a caution period, we need to update the Correctness property: the validity of the signature must be established at the signing date shifted (in the future) by the caution period:

Property 3 (Correctness with caution period). Given $S_{d}=\operatorname{Sig}(B, m, s a, s v)$, a caution period $C$, and Dispute $\left(A, B, S_{d}, T_{d}\right)$; if $\operatorname{RDR}\left(S_{d}, \mathcal{O}, \mathcal{I} \mathcal{V} \mathcal{T}(T), T_{d}, T\right)$ outputs true then $S_{d}$ is created at a date $T_{s} \leq T_{d}$ and is valid at $T_{s}+C$.

We also need to update the RDR semantics. The first statement in Definition 5 needs to be updated to the following: there exists a date $T_{x} \geq T_{s}+C$ such that $\operatorname{SVF}\left(S_{d}, \mathcal{K}, \mathcal{O}, T_{x}\right)=($ true,$P)$. For the sake of simplicity, the rest of the document will use the semantics without caution period. 


\section{Calculus for Long-Term Signature Validation}

This section defines a formal calculus modeling the resolution of dispute. It uses a simplified subset of the sequent logic syntax which provides a formal setting to reason about logical truth based on deduction using inference rules. The formulas are a set of statements indicating trust relationship. Axioms are a set of particular statements, called Judge's Initial View, accepted as trustworthy by the Judge prior to deriving any judgments. In contrast with classical sequent calculus, values assigned to formulas are not true and false, but valid and invalid. For instance, an invalid formula is not necessary false but indicates that the Judge does not trust the statement inferred from this formula.

\subsection{Statements}

The statements use date instants $T$ (now() will denote the current date) and date intervals (e.g. $I=\left[T_{b}, T_{e}\right]$ ). All validation objects considered below are signed objects. In general, a validation object may be denoted $V$ or $V(s a)$ where $s a$ is the signature algorithm used to $\operatorname{sign} V$. We will consider two types of statements:

Validation Object Statements. A validation object statement indicates that the Judge holds a validation object:

- $\operatorname{Sig}(X, m, s a, s v)$ denotes that the Judge holds a signature signed by $X$. The signature value $s v$ is computed on $m$ using the signature algorithm $s a$.

- $\operatorname{EECert}(X, Y, I, s a)$ denotes that the Judge holds an end entity certificate for $Y$ 's public key signed by $X$ using $s a$. The certificate is valid in $I$.

- CACert $(X, Y, I, s a)$ denotes that the Judge holds a CA's certificate for $Y$ 's public key signed by $X$ using $s a$. The certificate is valid in $I$.

- NRev $(X, Y, I, s a)$ denotes that the Judge holds an evidence signed by $X$ using $s a$ and indicating that $Y$ 's key is not revoked in $I$.

- TSP $\left(X, h(m), T_{t s p}, s a\right)$ denotes that the Judge holds a time-stamp signed by $X$ at $T_{t s p}$ using the signature algorithm $s a$ on the hash of $m$ using the cryptographic hash function $h$.

Assertion Statements. An assertion statement indicates that the Judge believes that an assertion is trustworthy:

- CAAut $(X, I)$ denotes that the Judge believes that $X$ is a CA and that $X$ 's public key is authentic in $I$.

- $\operatorname{Aut}(X, I)$ denotes that the Judge believes that $X$ is an entity which is not a CA and that $X$ 's public key is authentic in $I$.

- $P O E\left(V, T_{\text {poe }}\right)$ denotes that the Judge believes in the existence of the validation object $V$ at $T_{\text {poe }}$.

- POI $\left(V(s a), T_{\text {poe }}\right)$ denotes that the Judge believes in the existence of the validation object $V(s a)$ at $T_{\text {poe }}$ and that $V(s a)$ has not been forged.

- $\operatorname{Algo}\left(a, T_{a}\right)$ denotes that the Judge believes that the algorithm $a$ is trusted to be secure until at least $T_{a}$. 


\subsection{Initial View Construction}

The Initial View $I V$ is the set of initial statements considered trustworthy.

Definition 6 (Initial View Construction). Given the trust anchors $\mathcal{K}=\left\{\right.$ Cert $_{1}, \ldots$, Cert $\left._{n}\right\}$, the algorithm assertions $\mathcal{A}=\left\{\left(a_{1}, T_{a_{1}}\right), \ldots,\left(a_{p}, T_{a_{p}}\right)\right\}$, the signed validation objects $\mathcal{O}=\left\{V_{1}, \ldots, V_{k}\right\}$ and the signature $S=\operatorname{Sig}(B, m$, sa, sv); the initial view $I V=I V(\mathcal{K}, \mathcal{A}, \mathcal{O}, S)$ is constructed as follows:

1. initialize IV with the empty set.

2. if $S$ is cryptographically valid, add $S$ to $I V$.

3. $\forall i \in[1, k]$, if the signature in $V_{i}$ is cryptographically valid, add $V_{i}$ to $I V$.

4. For each certificate Cert $_{i}$ in $\mathcal{K}$, add CAAut $\left(X_{i}, I_{X_{i}}\right)$ to IV such that $X_{i}$ is the subject of the certificate Cert $t_{i}$ and $I_{X_{i}}$ is its validity interval.

5. For each pair $\left(a_{i}, T_{a_{i}}\right)$ in $\mathcal{A}$, add $\operatorname{Algo}\left(a_{i}, T_{a_{i}}\right)$ to $I V$.

\subsection{Inference Rules}

In our model, we will consider the following inference rules:

Authenticity of public keys. Informally, $Y$ is authentic if and only if the Judge holds a certificate for $Y$ signed by $X$ believed to be authentic and owned by a CA. Besides, the Judge must hold a revocation signed by $X$ indicating that $Y$ is not revoked. Finally, the judge must hold a $P O I$ for any used object. If $Y$ is a CA, the Judge derives $C A A u t$, otherwise, he derives Aut. $\forall T \in I=$ $I_{1} \cap I_{2} \cap I_{3}=\left[T_{1}, T_{2}\right]:$

$$
\begin{gathered}
C A A u t\left(X, I_{1}\right) \\
\frac{P O I\left(C A C e r t\left(X, Y, I_{2}, s a_{2}\right), T\right) \quad P O I\left(N \operatorname{Rev}\left(X, Y, I_{3}, s a_{3}\right), T\right)}{C A A u t\left(Y,\left[T, T_{2}\right]\right)} \\
\frac{P O I\left(E E C e r t\left(X, Y, I_{2}, s a_{2}\right), T\right) \quad P O I\left(N \operatorname{Rev}\left(X, Y, I_{3}, s a_{3}\right), T\right)}{A u t\left(Y,\left[T, T_{2}\right]\right)}
\end{gathered}
$$

Immediate proof of existence. Informally, if the Judge holds a validation object $V$, then he holds a proof of existence of this object at the current date.

$$
\frac{V}{\operatorname{POE}(V, \operatorname{now}())}
$$

Proof of existence based on time-stamps. In our model, this is the only way to derive a proof of existence of an object at a date in the past. $\forall T \in I_{X}$ :

$$
\frac{P O I\left(T S P\left(X, h(V), T_{T S P}, s a_{t s p}\right), T\right) \quad \operatorname{Aut}\left(X, I_{X}\right)}{\operatorname{POE}\left(h(V), T_{T S P}\right)}
$$




\section{Indirect proof of existence}

Theorem 1. If $m=h(d)$ is proven to exist at $T_{1}$ where $d$ is a message proven to exist at a date $T \geq T_{1}$ and $h$ is a preimage-resistant hash function secure until at least a date $T_{h} \geq T$, then $d$ is indirectly proven to exist at $T_{1}$.

The proof is straightforward and is relative to the resistance to preimage of the cryptographic hash function $h$ [15]. Indeed, this guarantees that, given $m$, until the date $T_{h}$ it is hard to find a message $d^{\prime}$ such that $m=h\left(d^{\prime}\right)$. This means that $d$ exists at $T_{1}$. This gives the following inference rule: $\forall T_{1} \leq T \leq T_{h}$ :

$$
\frac{\operatorname{POE}\left(h(V), T_{1}\right) \quad \operatorname{POE}(V, T) \quad \operatorname{Algo}\left(h, T_{h}\right)}{\operatorname{POE}\left(V, T_{1}\right)}
$$

Proof of integrity. Informally, this rule means that if a signed object exists at $T$ and the signature algorithm is trusted until at least $T_{s a} \geq T$, then the object exists at $T$ and has not been forged at $T . \forall T \leq T_{s a}$ :

$$
\frac{\operatorname{POE}(V(s a), T) \quad \operatorname{Algo}\left(s a, T_{s a}\right)}{\operatorname{POI}(V(s a), T)}
$$

Eternal proof of integrity. Informally, this rule means that if a signed object has not been forged at $T_{0}$, then the signed object has not been forged for any later date. $\forall T \geq T_{0}$ :

$$
\frac{P O I\left(V(s a), T_{0}\right)}{P O I(V(s a), T)}
$$

\subsection{Statement Validity}

Definition 7. Given an initial view $I V$, a statement $G$ is said valid at $T$ if and only if $G \in I V$ or $G$ can be derived at $T$ by using the inference rules.

Definition 8 (Derivation tree and derivation proof). Given an initial view $I V$ and a statement $G$ valid at $T$, the derivations used to derive $G$ from $I V$ can be represented by a tree rooted by $G$, where the leaves are statements from $I V$ and each non-leaf node is derived from its children using one inference rule. This tree is called the derivation tree of $G$ at $T$. The set of leaf nodes is called derivation proof of $G$ at $T$ and is denoted $P(I V, G, T)$.

Note that there may be several derivation trees for the same $G, I V$, and $T$ which may yield different derivation proofs. A derivation proof is only unique given a particular derivation tree. Intuitively, a proof $\mathcal{P}$ is a minimal subset of $I V$ that can allow the derivation of $G$ at $T$ using the same tree (or possibly another tree). 


\section{Implementing the Resolution of Dispute Rule}

This section uses the calculus defined above to implement the RDR semantics. First we define an additional assertion statement: $\operatorname{Res}_{A, B}(\operatorname{Sig}(B, m, s a, s v), T)$ denotes that the Judge believes in the validity of Alice's claims for the dispute $\operatorname{Dispute}(A, B, \operatorname{Sig}(B, m, s a, s v), T)$. The RDR semantics can be written using the following inference rule: $\forall T \geq T_{s}$ such that $T_{s} \leq T_{B_{2}}$ :

$$
\frac{\operatorname{POI}\left(\operatorname{Sig}(B, m, s a, s v), T_{s}\right) \quad \operatorname{Aut}\left(B,\left[T_{B_{1}}, T_{B_{2}}\right]\right)}{\operatorname{Res}_{A, B}(\operatorname{Sig}(B, m, s a, s v), T)}
$$

Definition 9 (Resolution of Dispute Rule). Given $\mathcal{I} \mathcal{T}(T)=(\mathcal{K}, \mathcal{A})$, the signature $S_{d}=\operatorname{Sig}(B, m, s a, s v)$, the set of the validation objects $\mathcal{O}$ provided by Alice and the Dispute $\left(A, B, S_{d}, T_{d}\right) ; \operatorname{RDR}\left(S_{d}, \mathcal{O}, \mathcal{I} \mathcal{V} \mathcal{T}(T), T_{d}, T\right)$ outputs true if and only if the statement $\operatorname{Res}_{A, B}\left(S_{d}, T_{d}\right)$ is valid at $T$ with the initial view $I V=I V\left(\mathcal{K}, \mathcal{A}, \mathcal{O}, S_{d}\right)$. In addition, if the Judge rules in favor of Alice, then $P\left(I V, \operatorname{Res}_{A, B}\left(S_{d}, T_{d}\right), T\right)$ is a judgment proof.

Proposition 2 (Correctness). Given a dispute Dispute $\left(A, B, S_{d}, T_{d}\right)$; the Judge rules in favor of Alice at $T$ according to Definition 5 if and only if he rules in favor of Alice at $T$ according to Definition 9.

The proof is depicted in Appendix A. This proposition will allow us to define some properties of the judgment proof using the calculus.

\subsection{Expiration of the Judgment Proof}

Since derivations are deterministic, if the derivation of $\operatorname{Res}_{A, B}\left(S_{d}, T_{d}\right)$ from $I V$ yields the derivation proof $\mathcal{P}$ then $\operatorname{Res}_{A, B}\left(S_{d}, T_{d}\right)$ can always be derived from $\mathcal{P}$ at $T$ by building the same derivation tree. Therefore, replaying the RDR at the same judgment date using as input the judgment proof gives the same result. An important question is the following: if Alice comes later with this proof, will the Judge also rule in her favor? Each statement in the proof has an expiration date. For instance, certificate, revocation and authenticity statements have a validity interval from which we have the expiration date. An algorithm trust statement has also an expiration date which is the expiration date of the algorithm.

Definition 10 (Expiration date of a proof). Given a judgment proof $\mathcal{P}_{p}$ obtained at a date $T_{p}$, let us denote $E_{1} \ldots E_{n}$ the expiration dates extracted from the statements in $\mathcal{P}$. The expiration date of $\mathcal{P}$ is denoted $E(\mathcal{P})$ and is defined by the following date:

$$
E(\mathcal{P})=\min \left\{E_{i}, E_{i}>T_{p}\right\}_{1 \geq i \geq n}
$$

Proposition 3. Given a proof $\mathcal{P}_{p}$ obtained at $T_{p}$ for $\operatorname{Dispute}\left(A, B, S_{d}, T_{d}\right)$; if at $T \in\left[T_{P}, E\left(\mathcal{P}_{p}\right)\right]$, Alice provides $\mathcal{O}$ such that $\mathcal{P}_{p} \subseteq I V\left(\mathcal{I} \mathcal{V} \mathcal{T}(T), \mathcal{O}, S_{d}\right)$, then the Judge will rule in favor of Alice for Dispute $\left(A, B, S_{d}, T\right)$ at $T$. In addition, if $T_{d}<T_{p}$, the Judge will rule in favor of Alice for Dispute $\left(A, B, S_{d}, T_{d}\right)$ at $T$. 
The proof is depicted in Appendix B. Therefore, Alice has interest to archive the validation objects in $\mathcal{P}_{p}$. During $\left[T_{p}, E\left(\mathcal{P}_{p}\right)\right]$, favorable judgments are guaranteed to Alice provided she comes with these objects. Note that this works because we assumed that any update to $\mathcal{I} \mathcal{V} \mathcal{T}$ guarantees that any assertion statement derived from $\mathcal{I} \mathcal{V} \mathcal{T}\left(T_{p}\right)$ can be derived from any later $\mathcal{I} \mathcal{V} \mathcal{T}(T)$. If Alice needs favorable judgments beyond $E\left(\mathcal{P}_{p}\right)$, she needs to renew this proof. The proposition below is the foundation of renewal algorithms that will be showed in the next section.

Proposition 4. Given a proof $\mathcal{P}_{p}$ obtained at $T_{p}$ for $\operatorname{Dispute}\left(A, B, S_{d}, T_{d}\right)$; let us denote $\left\{V_{1} \ldots V_{n}\right\}$ the subset of validation objects in $\mathcal{P}_{p}$. If at $T$, Alice provides $\mathcal{O}$ such that $\mathcal{P}_{p} \subseteq I V\left(\mathcal{I} \mathcal{T}(T), \mathcal{O}, S_{d}\right)$ and that the Judge can derive $\left\{\operatorname{POE}\left(V_{1}, T_{\text {poe }}\right) \ldots \operatorname{POE}\left(V_{n}, T_{\text {poe }}\right)\right\}$ where $T_{\text {poe }} \in\left[T_{p}, E\left(\mathcal{P}_{p}\right)\right]$, then the Judge will rule in favor of Alice for the Dispute $\left(A, B, S_{d}, T_{\text {poe }}\right)$ at $T$. In addition, if $T_{d}<T_{p}$, the Judge will rule in favor of Alice for the Dispute $\left(A, B, S_{d}, T_{d}\right)$ at $T$.

This is a corollary of Proposition 3 and the proof is omitted.

Example. We consider the Dispute $\left(A, B, S_{d}\right.$, now ()$)$ where $S_{d}=$ $\operatorname{Sig}(B, m, s a, s v)$. The RDR is executed at now() with the following: $\quad\left\{S_{d}, \quad E \operatorname{ECert}\left(X, B, I_{b}, s a\right), \quad N \operatorname{Rev}\left(X, B, I_{r b}, s a\right)\right\} . \quad$ We will use $\mathcal{I V T}($ now ()$)=\left(\left\{C A A u t\left(X, I_{x}\right)\right\},\left\{A l g o\left(\right.\right.\right.$ sa,$\left.\left.\left.T_{s a}\right)\right\}\right)$. We assume: $I_{r b}=\left[T_{1}, T_{2}\right]$ and now ()$\in I_{r b} \subset I_{b} \subset I_{x}$ and $T_{s a}$ is greater than the upper bound of $I_{x}$. First we build $I V$ (assuming that all objects have cryptographically valid signatures): $\left\{\operatorname{Sig}(B, m, s a, s v), \operatorname{EECert}\left(X, B, I_{b}, s a\right), N \operatorname{Rev}\left(X, B, I_{r b}, s a\right), \operatorname{Algo}\left(s a, T_{s a}\right)\right.$,

$\left.C A A u t\left(X, I_{x}, s a\right)\right\}$. From this $I V$, we can build the proof tree depicted in Figure 2. Leaf $P O I$ s are derived by successive application of Rules (3) and (6).

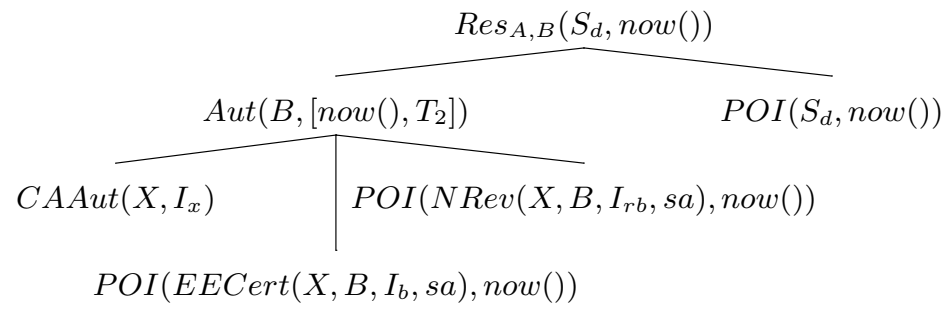

Fig. 2. Resolution proof tree

The judgment proof is: $\mathcal{P}=\left\{S_{d}, \operatorname{Aut}\left(X, I_{x}\right), \operatorname{EECert}\left(X, B, I_{b}, s a\right)\right.$, $\left.N \operatorname{Rev}\left(X, B, I_{r b}, s a\right), \operatorname{Algo}\left(s a, T_{s a}\right)\right\}$. This proof expires at $E(\mathcal{P})=T_{2}$.

\section{Using the Calculus in the Real World}

One of the motivations of this work is to propose a calculus that can be used to model several frameworks [4, 5, 6] used in practice for long-term signature 
validation. Most of theses frameworks rely on the use of ERS [8]. In this section, we will model the use of ERS to renew the validity of a signature beyond the expiration date of a judgment proof. For this purpose, we will model the following operations: ERS creation, simple renewal and complex renewals.

ERS Creation. Prior to create an ERS, Alice should ensure that she has appropriate validation data to have a favorable judgment at the current date for Dispute $\left(A, B, S_{d}\right.$, now ()$)$. With our calculus, this means that executing RDR at now () outputs a proof $\mathcal{P}_{0}$. The objective of Alice is to continuously renew this proof to guarantee favorable judgments for the dispute $\operatorname{Dispute}\left(A, B, S_{d}, T_{0}\right)$ where $T_{0}$ is as close as possible to the current date. For this purpose, Alice needs to create an ERS as soon as possible (before the date $E\left(\mathcal{P}_{0}\right)$ ).

ERS creation consists in requesting a time-stamp on the validation objects used to validate the signature. In our calculus, this means requesting timestamps for the validation objects $\left\{V_{1} \ldots V_{n}\right\}$ in $\mathcal{P}_{0}$. Let us denote $E R S_{0}=$ $\left\{T S P_{0} \ldots T S P_{n}\right\}$ the obtained time-stamps using the authority $X_{0}$, the cryptographic function $h_{0}$ and the signature algorithm $s a_{0}$.

Now, how $E R S_{0}$ can allow to have a favorable judgment after $E\left(\mathcal{P}_{0}\right)$ ? At $T>$ $E\left(\mathcal{P}_{0}\right)$, in addition to the validation objects $V_{1} \ldots V_{n}$, Alice needs to come with the set $E R S_{0}$ and the validation data that allow the Judge to derive $\operatorname{Aut}\left(X_{0}, I_{X_{0}}\right)$ at $T$. In this case, the Judge can derive at $T$, the statements $\operatorname{POE}\left(V_{i}, T_{0}\right)$ for any $i \in[1, n]$ using the tree in Figure 3. Note that this requires that $h_{0}$ and $s a_{0}$ are trusted at $T$. Thus, he will rule in favor of Alice for $\operatorname{Dispute}\left(A, B, S_{d}, T_{0}\right)$ (Proposition 4) with a judgment proof $\mathcal{P}_{1}$. During the interval $\left[T, E\left(\mathcal{P}_{1}\right)\right], \mathcal{P}_{1}$ guarantees favor judgments for Alice for $\operatorname{Dispute}\left(A, B, S_{d}, T_{0}\right)$. To go beyond $E\left(\mathcal{P}_{1}\right)$, Alice needs to continuously execute the renewal algorithms.

Simple Renewal. The simple renewal applies to the last time-stamps. i.e. the preceding time-stamps are time-stamped again together with complementary validation objects if needed. In our model, the simple renewal consists in requesting new time-stamps $\left(E R S_{1}\right)$ for the time-stamps in $E R S_{0}$ and any validation object used to derive $\operatorname{Aut}\left(X_{0}, I_{X_{0}}\right)$. This renewal must occur at $T_{1}<E\left(\mathcal{P}_{1}\right)$.

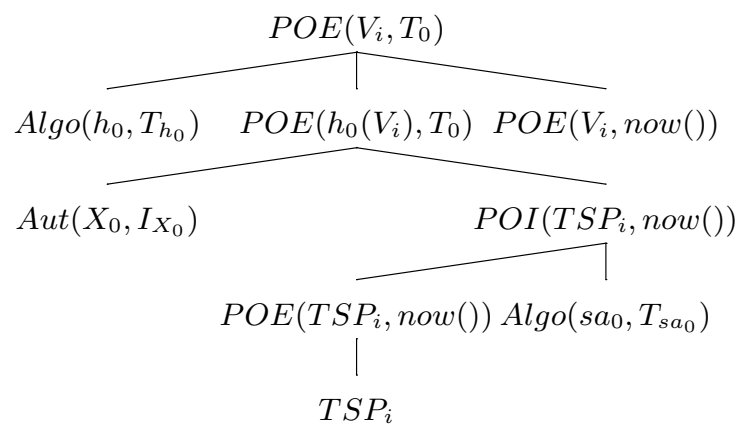

Fig. 3. Derivation of $\operatorname{POE}\left(V_{i}, T_{0}\right)$ after ERS creation $(T=$ now ()$)$ 
Now, how $E R S_{1}$ allows the Judge to rule in favor of Alice after $E\left(\mathcal{P}_{1}\right)$ ? Let denote $X_{1}, s a_{1}$ and $h_{1}$ respectively the TSA, the signature algorithm and the cryptographic hash function used at $T_{1}$. To have favorable judgments at a date $T>E\left(\mathcal{P}_{1}\right)$, Alice needs to provide $\left\{V_{1} \ldots V_{n}\right\}, E R S_{0}, E R S_{1}$ and the validation objects that allow to derive $\operatorname{Aut}\left(X_{1}, I_{X_{1}}\right)$ at $T$. From $E R S_{1}$ and the derivation proof of $\operatorname{Aut}\left(X_{1}, I_{X_{1}}\right)$, the Judge can derive $\operatorname{POE}\left(T S P\left(X_{0}, h_{0}\left(V_{i}\right), T_{0}, s a_{0}\right), T_{1}\right)$ for any $i \in[1, n]$ in the same way as in Figure [3. Also, since $E R S_{1}$ includes time-stamps on the validation objects used to derive the authenticity of $X_{0}$, this allows to derive $\operatorname{Aut}\left(X_{0}, I_{X_{0}}\right)$ at $T$. From that, the Judge can derive $\operatorname{POE}\left(V_{i}, T_{0}\right)$ for any $i \in[1, n]$ and therefore he will rule in favor of Alice (Proposition 4) using a proof $\mathcal{P}_{2}$. Note again that this requires that $h_{0}, h_{1}$, and $s a_{1}$ are trusted at $T$. $\mathcal{P}_{2}$ guarantees favorable judgments to Alice until the date $E\left(\mathcal{P}_{2}\right)$. Before this date, Alice needs to run again the simple renewal algorithm.

Recursive simple renewals may allow Alice to postpone the expiration of the judgment proof. Naturally, Alice should use state of art algorithms and authorities at each renewal to be able to validate the last time-stamps. However, recursive simple renewals can not allow "eternal" validity. Indeed, for all the proofs $\mathcal{P}_{i}$ obtained after $k$ simple renewals, $\operatorname{Algo}\left(h_{j}, T_{h_{j}}\right) \in \mathcal{P}_{i}, \forall j \leq i$ (where $h_{j}$ is the hash function used for the $j$ th simple renewal). Thus, for all the proofs $\mathcal{P}_{i}$ we have $E\left(\mathcal{P}_{i}\right)<\min \left\{T_{h_{j}}, j \leq i\right\}$. We can fairly assume that, at each renewal, Alice uses an up to date hash function such that $T_{h_{j+1}} \geq T_{h_{j}} \forall j$. Therefore, $T_{h_{0}}$ represents the maximum date for which Alice can have favorable judgments by recursive simple renewals. To have favorable judgments beyond this date, Alice needs to execute the complex renewal.

Complex Renewal. This renewal is performed when the probability of hash function compromise is foreseen. Not only time-stamps are re-timestamped but also data objects protected by the time-stamps. In our model, the need to execute this renewal occurs when the expiration date of a judgment proof $\mathcal{P}_{k+1}$ obtained after the $k$ th simple renewal becomes close to the lowest expiration date (say $T_{h_{0}}$ ) of the hash functions used in the previous simple renewals. The renewal must be executed at a date $T_{k+1}<E\left(\mathcal{P}_{k+1}\right)$. It consists in creating a new ERS on: $\left\{V_{1}, \ldots, V_{n}\right\}, E R S_{k}$ and the objects used at $T_{k+1}$ to derive $\operatorname{Aut}\left(X_{k}, I_{X_{k}}\right)$ where $X_{k}$ is the TSA used for the $k$ th simple renewal.

Now, how the complex renewal allows the Judge to rule in favor of Alice at a date $T>T_{h_{0}}$ ? Let us denote $h_{k+1}, X_{k+1}$ and $E R S_{k+1}$ respectively the hash function, the TSA and the set of obtained time-stamps. At $T$, Alice needs to come with the validation objects in $\mathcal{P}_{k+1}, E R S_{k+1}$ and the objects that allow to derive $\operatorname{Aut}\left(X_{k+1}, I_{X_{k+1}}\right)$. Since the complex renewal is also a simple renewal, it allows to derive $\operatorname{POE}\left(T S P\left(X_{0}, h_{0}\left(V_{i}\right), T_{0}, s a_{0}\right), T_{1}\right)$ for any $i$ in $[1, n]$ and $\operatorname{Aut}\left(X_{0}, I_{X_{0}}\right)$ at $T$. In addition, $E R S_{k+1}$ includes a time-stamp on the object $V_{i}$ which allows to derive $\operatorname{POE}\left(V_{i}, T_{k+1}\right)$ for any $i$ in $[1, n]$. From that, $\operatorname{POE}\left(V_{i}, T_{0}\right)$ can be derived at $T$ for any $i \in[1, n]$. Thus, the Judge will rule in favor of Alice at $T$ (Proposition 4) with a proof $\mathcal{P}_{k+2}$. If Alice needs favorable judgments after $E\left(\mathcal{P}_{k+2}\right)$, she needs to continue to execute the simple renewal. Again, once the 
expiration date of a proof becomes close to the expiration of the hash functions used with a previous ERS, the complex renewal should be performed.

\section{Conclusion and Future Work}

We presented a security model for digital signature validation with the notion of dispute about the validity of a signature. The first contribution of this paper is the definition of the semantics of a resolution of dispute rule. The second contribution is a new calculus for reasoning about the validation of digital signatures at a particular date which may be in the past. The usefulness of our calculus is demonstrated through modeling ERS, one of the main techniques used in practice. The calculus allowed to model at which date the renewal becomes necessary, which data must be archived and how the renewal allows to have a favorable judgment after the expiration of the last proof.

One of the main components of the model is $\mathcal{I} \mathcal{V} \mathcal{T}$. The hypothesis that all actors trust a common set of trust anchors and algorithms seems very strong. If we can establish such agreement in a non-repudiable way, one may ask why this same way is not used to implement the resolution of dispute. On the other hand, this agreement requires that Bob agrees on the content of $\mathcal{I} \mathcal{V} \mathcal{T}$ at the signing date. We may assume that this $\mathcal{I V} \mathcal{T}$ is part of the signed message which proves Bob's commitment. However, because we need to continuously update $\mathcal{I} \mathcal{V} \mathcal{T}$, we need Bob's commitment for each new $\mathcal{I} \mathcal{V} \mathcal{T}$. Bob's collaboration is then necessary for long-term validation which may be refused by Bob. In real world, this issue is resolved by the use of a third trusted party. The origin of this trust is usually legal frameworks [3, 16, 17] to which all other actors refer in case of dispute.

An interesting future work is the definition of other semantics for RDR. Ideally, in the scope of long-term validation, completely honest behaviors should not be an assumption. A potential interesting work is to capture other PKI models with potential malicious actors. This suggests the extension of the calculus to a model based on confidence valuation similar to the probabilistic model proposed by Maurer [9]. This will allow to define an evaluation metric for the judgment proofs based on the confidence levels of the statements composing the proofs. The Judge would select the proof with the highest confidence level and rule in favor of Alice provided this level goes beyond a determined threshold.

Acknowledgment. The authors would like to thank the anonymous reviewers for their valuable comments.

\section{References}

1. Cooper, D., Santesson, S., Farrell, S., Boeyen, S., Housley, R., Polk, W.: Public key infrastructure: Certificate and CRL profile, IETF, Tech. Rep. RFC 5280 (2008)

2. Aarnes, A.: Public key certificate revocation schemes, Ph.D. dissertation, Norwegian University of Science (2000)

3. ETSI, Algorithms and parameters for secure electronic signatures; part 1: Hash functions and asymmetric algorithms, Tech. Rep. ETSI TS 102 176-1 V2.0.0 (2007) 
4. Jerman Blaič, A., Klobučar, T., Jerman, B.D.: Long-term trusted preservation service using service interaction protocol and evidence records. Comput. Stand. Interfaces 29 (2007)

5. Huhnlein, D., Korte, U., Langer, L., Wiesmaier, A.: A comprehensive reference architecture for trustworthy long-term archiving of sensitive data. In: Third International Conference on New Technologies, Mobility and Security (2009)

6. Troncoso, C., De Cock, D., Preneel, B.: Improving secure long-term archival of digitally signed documents. In: Proceedings of the 4th ACM International Workshop on Storage Security and Survivability, pp. 27-36. ACM, New York (2008)

7. ETSI, CMS Advanced Electronic Signatures, Tech. Rep. ETSI TS 101733 (2008)

8. Gondrom, T., Brandner, R., Pordesch, U.: Evidence Record Syntax (ERS), Tech. Rep. RFC4998 (2007)

9. Maurer, U.M.: Modelling a public-key infrastructure. In: Martella, G., Kurth, H., Montolivo, E., Hwang, J. (eds.) ESORICS 1996. LNCS, vol. 1146, pp. 325-350. Springer, Heidelberg (1996)

10. Marchesini, J., Smith, S.: Modeling public key infrastructures in the real world. In: Chadwick, D., Zhao, G. (eds.) EuroPKI 2005. LNCS, vol. 3545, pp. 118-134. Springer, Heidelberg (2005)

11. Bicakci, K., Crispo, B., Tanenbaum, A.S.: How to incorporate revocation status information into the trust metrics for public-key certification. In: Proceedings of the 2005 ACM Symposium on Applied Computing, pp. 1594-1598. ACM, New York (2005)

12. Baier, H., Karatsiolis, V.: Validity models of electronic signatures and their enforcement in practice. In: Martinelli, F., Preneel, B. (eds.) EuroPKI 2009. LNCS, vol. 6391, pp. 255-270. Springer, Heidelberg (2010)

13. Goldwasser, S., Micali, S., Rivest, R.L.: A digital signature scheme secure against adaptive chosen-message attacks. SIAM Journal on Computing 17, 281-308 (1988)

14. Walleck, D., Li, Y., Xu, S.: Empirical analysis of certificate revocation lists. In: Atluri, V. (ed.) DAS 2008. LNCS, vol. 5094, pp. 159-174. Springer, Heidelberg (2008)

15. Menezes, A.J., van Oorschot, P.C., Vanstone, S.A.: Handbook of Applied Cryptography. Discrete Mathematics and Its Applications. CRC Press, Boca Raton (1997)

16. FNISA, Annexe B1 - règles et recommandations concernant le choix et le dimensionnement des mécanismes cryptographique, French Network and Information Security Agency, Tech. Rep. Version 1.20 du (janvier 26, 2010)

17. ETSI, Provision of harmonized trust service provider status information, Tech. Rep. ETSI TS 102231 V3.1.2 (2009)

\section{A Proof of Proposition 2}

Let us first assume that the Judge rules in favor of Alice according to Definition 5. We want to prove that with the same inputs, the Judge will rule in favor of Alice according to Definition [9]. Definition 5 means that there exists a date $T_{s} \leq T_{d}$ such that the three statements of the definition are satisfied at $T$.

1. The first statement means that there exists a date $T_{x} \geq T_{s}$ such that $\operatorname{SVF}\left(S_{d}, \mathcal{K}, \mathcal{O}, T_{x}\right)=($ true,$P)$, where $P$ is a sequence of certificates and revocation objects. Let us denote this sequence by: 


$$
P=\left(\begin{array}{c}
C A C \operatorname{Cert}\left(X_{0}, X_{1}, I_{1}, s a_{1}\right), N \operatorname{Rev}\left(X_{0}, X_{1}, I_{1}^{\prime}, s a_{1}^{\prime}\right) \\
\cdots \\
C A \operatorname{Cert}\left(X_{n-2}, X_{n-1}, I_{n-1}, s a_{n-1}\right), N \operatorname{Rev}\left(X_{n-2}, X_{n-1}, I_{n-1}^{\prime}, s a_{n-1}^{\prime}\right) \\
\operatorname{EE\operatorname {Cert}}\left(X_{n-1}, X_{n}, I_{n}, s a_{n}\right), N \operatorname{Rev}\left(X_{n-1}, X_{n}, I_{n}^{\prime}, s a_{n}^{\prime}\right)
\end{array}\right)
$$

From the definition of $S V F$, we have the following:

$-X_{0}$ is a trust anchor from $\mathcal{K}$. Therefore, there exits an interval $I_{0}$ such that $T_{x} \in I_{0}$ and $C A A u t\left(X_{0}, I_{0}\right) \in I V$.

- the last certificate is issued to $\operatorname{Bob}\left(X_{n}=B\right)$.

- the signatures in $S_{d}$, the certificates and revocation objects in $P$ are cryptographically valid. Therefore, $\left\{S_{d}, P\right\} \subset I V$.

- all certificates and revocation objects in $P$ are valid at $T_{x}$. Therefore, $\forall i \in[1, n], T_{x}$ is included in $I_{i}$ and $I_{i}^{\prime}$.

2. The second statement means that any validation object in $P$ exists at a date earlier than $T_{x}$ and that at this date the signature algorithm used to protect the object is trusted to be secure. This gives us the following:

- $\forall i \in[0, n-2]$, there exists a date $T_{i} \leq T_{x}$ (respectively a date $T_{i}^{\prime} \leq T_{x}$ ) such that $\operatorname{POE}\left(C A C \operatorname{Cert}\left(X_{i}, X_{i+1}, I_{i+1}, s a_{i+1}\right), T_{i}\right)$ (respectively $\left.\operatorname{POE}\left(N \operatorname{Rev}\left(X_{i}, X_{i+1}, I_{i+1}^{\prime}, s a_{i+1}^{\prime}\right), T_{i}^{\prime}\right)\right)$ can be derived at $T$.

- There exists a date $T_{n} \leq T_{x}$ (respectively a date $T_{n}^{\prime} \leq T_{x}$ ) such that $\operatorname{POE}\left(\operatorname{EECert}\left(X_{n-1}, X_{n}, I_{n}, s a_{n}\right), T_{n}\right)$ (respectively $\left.\operatorname{POE}\left(N \operatorname{Rev}\left(X_{n-1}, X_{n}, I_{n}^{\prime}, s a_{n}^{\prime}\right), T_{n}^{\prime}\right)\right)$ can be derived at $T$.

$-\forall i \in[1, n]$, there exists a date $T_{s a_{i}} \geq T_{i}$ such that $\left(s a_{i}, T_{s a_{i}}\right)$ is in $\mathcal{A}$ and then $\operatorname{Algo}\left(s a_{i}, T_{s a_{i}}\right) \in I V$.

With theses POE and Algo statements, the Judge can derive the following statements using Rule (6) to have a $P O I$ with a date earlier than $T_{x}$ then Rule (7) to have a $P O I$ with $T_{x}$ :

- $\forall i \in[0, n-2]: P O I\left(C A C e r t\left(X_{i}, X_{i+1}, I_{i+1}, s a_{i+1}\right), T_{x}\right)$ and $\operatorname{POI}\left(N \operatorname{Rev}\left(X_{i}, X_{i+1}, I_{i+1}^{\prime}, s a_{i+1}^{\prime}\right), T_{x}\right)$

- POI $\left(E E C \operatorname{Cert}\left(X_{n-1}, X_{n}, I_{n}, s a_{n}\right), T_{x}\right)$

- POI $\left(N \operatorname{Rev}\left(X_{n-1}, X_{n}, I_{n}^{\prime}, s a_{n}^{\prime}\right), T_{x}\right)$

Now, we will show that, with the previous statements, the Judge can derive $\operatorname{Aut}\left(B, I_{B}\right)$ such that $I_{B}$ is an interval that contains the date $T_{x}$. First, let us prove by induction the following: $\forall i \in[1, n-1]$, there exists an interval $I_{i}^{\prime \prime}$ such that $T_{x} \in I_{i}^{\prime \prime}$ and the statement $C A A u t\left(X_{i}, I_{i}^{\prime \prime}\right)$ is valid at $T$ :

- for $i=1, C A A u t\left(X_{1}, I_{1}^{\prime \prime}\right)$ can be derived with the following such that $T_{x} \in I_{1}^{\prime \prime}$ (Rule (1)):

$$
\begin{gathered}
C A A u t\left(X_{0}, I_{0}\right) \quad \operatorname{POI}\left(C A C e r t\left(X_{0}, X_{1}, I_{1}, s a_{1}\right), T_{x}\right) \\
\operatorname{POI}\left(N \operatorname{Rev}\left(X_{0}, X_{1}, I_{1}^{\prime}, s a_{1}^{\prime}\right), T_{x}\right)
\end{gathered}
$$

This works because we showed that $C A A u t\left(X_{0}, I_{0}\right) \in I V$, and $T_{x}$ is included in all the intervals $I_{i}$ and $I_{i}^{\prime}$. 
- Now we assume that there exists an interval $I_{i}^{\prime \prime}$ such that $T_{x} \in I_{i}^{\prime \prime}$ and $C A A u t\left(X_{i}, I_{i}^{\prime \prime}\right)$ is valid at $T$. The application of Rule (1D) gives $C A A u t\left(X_{i+1}, I_{i+1}^{\prime \prime}\right)$ such that $T_{x} \in I_{i+1}^{\prime \prime}$ :

$$
\begin{gathered}
\operatorname{CAAut}\left(X_{i}, I_{i}^{\prime \prime}\right) \quad \operatorname{POI}\left(C A C \operatorname{Cert}\left(X_{i}, X_{i+1}, I_{i+1}, s a_{i+1}\right), T_{x}\right) \\
\operatorname{POI}\left(\operatorname{Nev}\left(X_{i}, X_{i+1}, I_{i+1}^{\prime}, s a_{i+1}^{\prime}\right), T_{x}\right)
\end{gathered}
$$

Therefore, there exists an interval $I_{n-1}^{\prime \prime}$ such that $T_{x} \in I_{n-1}^{\prime \prime}$ and $C A A u t\left(X_{n-1}, I_{n-1}^{\prime \prime}\right)$ is valid at $T$. Now, we use Rule (2) to derive $\boldsymbol{A u t}\left(\boldsymbol{B}, \boldsymbol{I}_{\boldsymbol{B}}\right)$ such that $T_{x} \in I_{B}$ :

$$
\begin{gathered}
\operatorname{CAAut}\left(X_{n-1}, I_{n-1}^{\prime \prime}\right) \\
P O I\left(N \operatorname{Rev}\left(X_{n-1}, X_{n}, I_{n}^{\prime}, s a_{n}^{\prime}\right), T_{x}\right)
\end{gathered}
$$

3. The last statement in Definition 5 gives the following: $\operatorname{POE}\left(S_{d}, T_{s}\right)$ is valid at $T$ and there exists a date $T_{s a} \geq T_{s}$ such that $\left(s a, T_{s a}\right)$ is in $\mathcal{A}$ and then in $\operatorname{Algo}\left(s a, T_{s a}\right) \in I V$. Therefore, Rule (6) allows to derive $\boldsymbol{P O I}\left(\boldsymbol{S}_{\boldsymbol{d}}, \boldsymbol{T}_{\boldsymbol{s}}\right)$.

In conclusion, we showed that the Judge can derive the statements $\operatorname{POI}\left(S_{d}, T_{s}\right)$ and $\operatorname{Aut}\left(B, I_{B}=\left[T_{B_{1}}, T_{B_{2}}\right]\right)$ at $T$ where $T_{d} \geq T_{s}$ and $T_{s} \leq T_{B_{2}}$ (because $T_{s} \leq T_{x}$ and $\left.T_{x} \in I_{B}\right)$. Then, Rule (8) allows to derive $\operatorname{Res}_{A, B}\left(S_{d}, T_{d}\right)$ at $T$. Therefore, the Judge will rule in favor of Alice according to Definition 9 .

The other part of the proof is similar and is omitted.

\section{B Proof of Proposition 3}

We want to prove that Alice will have a favorable judgment at any date $T \in$ $\left[T_{P}, E\left(\mathcal{P}_{p}\right)\right]$ provided she comes with the objects $\mathcal{O}$ such that $\mathcal{P}_{p} \subseteq I V=$ $I V\left(\mathcal{I} \mathcal{T}(T), \mathcal{O}, S_{d}\right)$. If the Judge rules in favor of Alice at $T_{p}$, this means that $\operatorname{Res}_{A, B}\left(S_{d}, T_{d}\right)$ can be derived at $T_{p}$ from $\mathcal{P}_{p}$. Then, there exist an interval $I_{B}=\left[T_{B_{1}}, T_{B_{2}}\right]$ and a date $T_{s}$ verifying $T_{s} \leq T_{B_{2}}$ and $T_{s} \leq T_{d}$ such that the statements $P O I\left(S_{d}, T_{s}\right)$ and $A u t\left(B, I_{B}\right)$ can be derived at $T_{p}$. Aut $\left(B, I_{B}\right)$ means that the Judge believes at $T_{p}$ that $B$ 's key is authentic within the interval $I_{B}$. Let us extract the expiration dates from the statements in $\mathcal{P}_{p}$ and order them chronologically in the following dates $E_{1} \ldots E_{k}$. With $T_{p}$, this gives us $k+2$ intervals. Within each interval, the validity status of all statements in $\mathcal{P}_{p}$ are constant. Therefore, the validity status of any Aut and $C A A u t$ is constant within each interval. We consider the date $E_{j}=E(\mathcal{P})=\min \left\{E_{i}, E_{i}>T_{p}\right\}_{1 \geq i \geq k}$. Since $\operatorname{Aut}\left(B, I_{B}\right)$ can be derived at $T_{p}$ from $\mathcal{P}_{p}$, then $\operatorname{Aut}\left(B, I_{B}\right)$ can be derived at any date $T \in\left[T_{p}, E\left(\mathcal{P}_{p}\right)\right]$. In addition, since $S_{d}$ and $\operatorname{Algo}\left(s a, T_{s a}\right)$ are in $\mathcal{P}_{p}$, then Rule (3) gives $\operatorname{POE}\left(S_{d}, T\right)$ and Rule (6) gives $\operatorname{POI}\left(S_{d}, T\right)\left(T \leq E\left(\mathcal{P}_{p}\right) \leq T_{s a}\right)$. Finally, Rule (8) gives $\operatorname{Res}_{A, B}\left(S_{d}, T\right)\left(T \leq E\left(\mathcal{P}_{p}\right) \leq T_{B_{2}}\right)$. Therefore, the Judge will rule in favor of Alice at $T$ for the dispute Dispute $\left(A, B, S_{d}, T\right)$. 


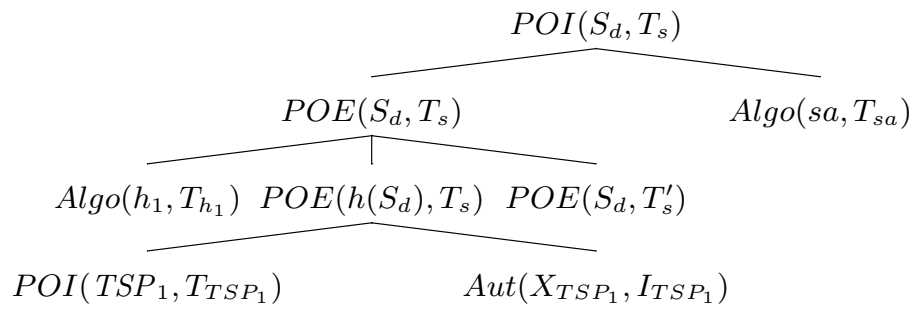

Fig. 4. Derivation of $\operatorname{POI}\left(S_{d}, T_{s}\right)$ at $T_{p}\left(T_{s} \leq T_{s}^{\prime} \leq T_{h_{1}}\right.$ and $\left.T_{T S P_{1}} \in I_{T S P_{1}}\right)$

\begin{tabular}{|c|c|c|}
\hline \multicolumn{3}{|c|}{$P O I\left(T S P_{1}, T_{T S P_{1}}\right)$} \\
\hline \multicolumn{2}{|r|}{$P O E\left(T S P_{1}, T_{T S P_{1}}\right)$} & $\operatorname{Algo}\left(s a_{1}, T_{s a_{1}}\right)$ \\
\hline $\operatorname{Algo}\left(h_{2}, T_{h_{2}}\right)$ & $P O E\left(h_{2}\left(T S P_{1}\right), T_{T S P_{1}}\right.$ & \\
\hline$P O I\left(T S P_{2}, T_{T S P}\right.$ & & \\
\hline
\end{tabular}

Fig. 5. Derivation of $P O I\left(T S P_{1}, T_{T S P_{1}}\right)$ at $T_{p}$

Now, we consider the case $T_{d}<T_{p}$. This means that at $T_{p}$, the Judge derived a $P O I$ for $S_{d}$ with a date in the past $\left(T_{s} \leq T_{d}<T_{p}\right)$. The only way is using Rule (4) with a time-stamp $T S P_{1}=T S P\left(X_{T S P_{1}}, h_{1}\left(S_{d}\right), T_{s}, s a_{1}\right)$ as in Figure 4. First, using the same reasoning as above for $\operatorname{Aut}\left(B, I_{B}\right), \operatorname{Aut}\left(X_{T S P_{1}}, I_{T S P_{1}}\right)$ can be derived at any date $T \in\left[T_{p}, E\left(\mathcal{P}_{p}\right)\right]$. Second, we have two cases:

- If $T_{T S P_{1}}=T_{p}$, then $\left[T_{p}, E\left(\mathcal{P}_{p}\right)\right] \subset I_{T S P_{1}}$ and therefore $T \in I_{T S P_{1}}$. Thus, $P O I\left(S_{d}, T_{s}\right)$ can be derived at $T$ with the derivation tree in Figure 4 using $T$ instead of $T_{T S P_{1}}$ and $T_{s}^{\prime}$ (recall that $T \leq E\left(\mathcal{P}_{p}\right) \leq T_{h_{1}}$ ).

- If $T_{T S P_{1}}<T_{p}$, then the Judge derived at $T_{p}$ a $P O I$ statement for $T S P_{1}$ with a date $T_{T S P_{1}}<T_{p}$. The only way is using Rule (4) with $T S P_{2}=$ $T S P\left(X_{T S P_{2}}, h_{2}\left(T S P_{1}\right), T_{T S P_{1}}, s a_{2}\right)$ (Figure 5$)$. We have again two cases:

- If $T_{T S P_{2}}=T_{p}$, then $P O I\left(T S P_{1}, T_{T S P_{1}}\right)$ can be derived at $T$ using $T_{T S P_{2}}=T_{T S P_{1}}^{\prime}=T$ and thus, $P O I\left(S_{d}, T_{s}\right)$ can be derived at $T$.

- If $T_{T S P_{2}}<T_{p}$, then $P O I\left(T S P_{2}, T_{T S P_{2}}\right)$ was derived at $T_{p}$ using Rule 4 with a time-stamp $T S P_{3}=T S P\left(X_{T S A_{3}}, h_{3}\left(T S P_{2}\right), T_{T S P_{2}}, s a_{3}\right)$.

Recursively, we end with a time-stamp $T S P_{n}$ such that $T_{T S P_{n}}=T_{p}$ and then $P O I\left(T S P_{n-1}, T\right), \ldots, P O I\left(T S P_{1}, T\right)$ can be derived at $T$ which allows to derive $\operatorname{POI}\left(S_{d}, T_{s}\right)$ at $T$.

Therefore, in both cases $P O I\left(S_{d}, T_{s}\right)$ can be derived at $T$. This allows the Judge to derive $\operatorname{Res}_{A, B}\left(S_{d}, T_{d}\right)$ at $T$ and then to rule in favor of Alice at $T$ for the dispute Dispute $\left(A, B, S_{d}, T_{d}\right)$. 